\title{
Some Problems in the Treatment of Puerperal Septic Diseases.
}

\author{
By A. Kryvett Gordox, M.B. (Cantab.), \\ Medical Superintendent of Monsall Hospital; Lecturer on Infectious \\ Diseases in the University of Manchester.
}

I propose here to mention some problems which the variety of wound infection that wo know as puerperal fever seems to propound for our solution. The following paper is based on some remarks which I made in introducing a discussion on the subject at a recent meeting of the North of England Obstetrical Society.

It will first be necessary to limit the field of view somewhat, and I can best do this by confining myself to curative treatmentomitting all questions connected with prophylaxis-and to that of the severe cases only, such, in fact, as have come under my own care at Monsall Hospital, where notified cases of this disease are regularly treated. I ought, perhaps, to mention that the extent of this experience is now some 250 consecutive cases, and that in each instance a bacteriological investigation of the nterine contents, and, when necessary, of the blood also, has been made in the hospital laboratory.

In the majority of the cases repeated blood counts have been made, and in some a detailed histological examination of the blood also. Postmortem examinations have been obtained in about 80 per cent. of the fatal cases, and whenever possible the distribution of the infecting organisms has been studied by large paraffin sections through the whole uterine wall.

In addition to the information obtained from personal observation, Dr. J. Niven, Medical Officer of Health, and Dr. Merry Smith, Medical Officer of the Midwives' Supervising Committee, have very courteously furnished me with historics of the confinements and of the events preceding the admission of the cases. I have also frequently had the advantage of personal conversation with the certifying medical attendant.

I will clear the ground still further by saying that I do not propose to discuss those cases in which there is an obvious local lesion calling equally clearly for surgical treatment on recognized lines, such, for instance, as cases of retention of fairly large portions of decomposing placental tissue, well-marked pelvic abscesses pointing in accessible regions, or where general suppurative peritonitis has supervened.

We are left then with the type of case in which the patient is desperately ill, where the extent of septic absorption is great, 
whether of toxins only or of bacteria also, and where there are no very obvious local signs, except uterine subinvolution, with, it may be, some abdominal distension, and endometritis. In practice about one-half of these have streptococci in the circulating blood at a fairly early date, other organisms - such as B. coli-being only found in that situation as a terminal invasion.

How are we to treat such cases clinically? There can be no doubt that the impression in the minds of most medical practitioners is that the one essential is an intra-uterine douche, and that this is generally supplemented by vaginal douching by the nurse. This teaching is undoubtedly to be found in the majority of textbooks on obstetries, the general impression to be obtained from which is that active disinfection of the uterus should be aroided. It is necessary to take this opinion very seriously, for it is held by men whose utterances must always command respect, and it is taught at this time to the great bulk of students in Great Britain and Ireland. The pathological foundation for this teaching is, I think, to be found in the teaching of Metchnikoff that between the system and the parasite lies a wall of leucocytic infiltration, and that we must not do anything that may impair the integrity of this structure, or we shall produce dissemination of the infecting organisms.

Another view is that we should treat the uterine wall as we should the surface of the lesion in any other variety of wound infection and disinfect it as best we ean. Let me for the moment take the analogy of a case of septic absorption from sloughing of the tonsils in the course of scarlet fever. Here, I imagine, there would be no doubt that we should do something more than wash the surface of the fauces with an antiseptic solution. We might, or might not serape the septic wound, but we should certainly rub in some strong disinfectant, nor should we be deterred from doing this by the belief that we were unable to reach all the organisms living in the depths of the part.

Similarly, those who counsel active interference in puerperal sepsis might or might not curette, but they would certainly rub in a powerful germicide, and would not be content with an intra-uterine douche. To the "active" school, the presence of puerperal septic disease of the type to which I am now alluding is, ipso facto, evidence that the granulation wall is usoless, or, as Sir William Sinclair has happily put it, has been breached or scaled.

On this point, I may perhaps be permitted to refer to my own experience, as it will account for the view which I hold. When I first began to treat these cases, I was influenced by what I had been taught, and had read, and contented myself with general measures, including the administration of antistreptococcic serim, and with intra-uterine and vaginal douches. Seventy-nine cases were treated this way and 37 died, a mortality of 46 per cent. Could any results be more disappointing? 
Furthermore, on examining the series of sections from the uterine wall of the fatal cases, I failed entirely to find a granulation wall at all in any instance. Both the "protective" leucocytes and the bacteria were scattered throughout the section from endometrium to peritoneum.

Then I began to disinfect, using a large sharp curette, followed by the application of undiluted izal fluid with a succession of swabs, with the result that, with no appreciable difference in the type of case admitted, the mortality fell to 24 per cont., where it has remained for three years. I must confess myself now unable to believe in the harmfulness of active local treatment, or to teach it. The first problem, therefore, whether it is right to continue to teach the policy of non-interference with the uterus in cases of general septicamia such as I have described.

Is it possible, however, that this teaching is founded on the results of curetting alone, or of curetting followed only by the douche? If so, there is every reason for dissatisfaction with the practice. As regards the use of the douche, the proportion of our cases in which both intra-uterine and vaginal douching had been followed before admission by steady deterioration in the condition of the patient is so high as to put the suggestion of mere coincidence quite out of court. I would make it clear that I regard intrauterine douching as more deleterious than vaginal irrigation, and it is difficult to avoid the conclusion that the former practice is responsible for a large number of avoidable deaths from puerperal sepsis.

The next point which I would raise is a difficulty presented by the treatment of the acute stage of pelvic cellulitis of puerperal origin. In quite a large number of the Monsall cases, especially where there has been a septic laceration of the cervix, a greater or less degree of inflammation of the pelvic connective tissue has been present on admission, and has, therefore, been one of the phenomena of the acute stage. In the large majority of these, there has not been at any time any evidence of suppuration, and in the usual course of events the swelling has subsided. The mischief, however, has been done. In some cases, pyæmia has supervened, and one has then been unable to discover, and, therefore, to treat the primary focus. 'This may, of course, have been the uterus itself, but when there has been co-existent parametritis, it is difficult to put this complication out of court as also a causative factor. In other words, because an acute pelvic cellulitis subsides without operative treatment, does it follow that we have done right in leaving it alone, or ought we to incise and pack and drain the inflamed area by an incision in the posterior cul de sac? And if we did, should we be more likely to avoid pyæmia? What will be the effect on the course of the attack? Here, I take it, we need not concern ourselves very much with the immediate effect of the operation, as a general 
anæsthetic is not necessary, and the procedure is not attended with any degree of shock.

It is necessary to point out that $I$ am referring to incision of the cellulitic area quite early in the attack and before there are any obvious signs of suppuration, also that the peritoneal cavity should not be opened.

Perhaps the most difficult cases that we have to treat at Monsall are those where the patient suffers from pyrexia prolonged for several weeks without any gross lesion other than an initial cellulitis to account for it. In many of these there is doubtless metro-phlebitis, with or without thrombosis of the veins in the broad ligament, and I am certainly of opinion that if this condition could be diagnosed wth anything approaching certainty we should be able to save the patient many weeks of illness by ligaturing these efferent veins. But the attempt to do this would certainly mean a very large number of purely exploratory operations on patients whose powers of resistance are not very great.

The next problem is whether hysterectomy in the acute stage has a legitimate place in the treatment of puerperal infection, and if so what are the indications for this radical measure.

Of the practice of removing the uterus quite early, before the patient is serionsly ill, I have had no experience, and I think I should, in any case, try active disinfection first. I have, however, performed the operation as a last resort in five cases, but never with success.

Leaving out these two extremes, however, I cannot but think that there are some cases where the infection of the uterus is intense, and local disinfection is inadequate, but where the organisms do not penetrate so rapidly as to make any operation hopeless. In such a case, there would be an appreciable interval, during which the process of toxin production was confined to the walls of the uterus, being hindered from spreading further, in all probability, by thrombosis of the veins in and around the organ itself. Unfortunately, there is a great difficulty clinically in detecting the existence of this stage, for by the time one is quite certain that local disinfection has failed, the mischief has usually spread beyond the uterus. In one of my own cases, however, the condition of the uterus, as observed at the time of the curetting, gave some indication that hysterectomy would probably be required. The case is an interesting one for many reasons, and will be published in extenso later on, but I may refer to it briefly in this connection.

The patient was seen for the first time on the third or fourth day of the pyrexia; when examined, the uterus was very soft, and it would have been possible to push the finger anywhere through its substance with the greatest ease. As there was also a large foetid mass of retained placental tissue, curetting and swabbing with izal were tried first. The next day there was no improvement, and I 
removed the uterus by the supra-vaginal operation, leaving one ovary. There was some trouble from sloughing of the retained cervix, but the pyrexia from this subsided, and the patient is now convalescent. On examination, the excised uterus was found to be practically gangrenous, and it is difficult to think that any measure of local disinfection could have sufficed.

Still, the worst cases are those where the uterus does not appear to be very much diseased. With the exception-though this is an important one- of extreme dilatation, and of a complete absence of any contraction after curetting and swabbing, there is no clinical evidence of local infection, but postmortem one finds such an organ packed with the infecting organism from peritoneum to endometrium. Probably in some cases one wastes valuable time in curetting, but it is difficult to accept the position that one should remove the uterus, say, when streptococci were found in its interior (but not in the circulating blood) and where no reaction to curetting was apparent, without operaling in many cases quite unjustifiably, and without a fatal issue in others from the shock of the procedure itself in a patient whose powers of resistance were at a very low ebb.

Suppuration in the wall of the uterus itself, whether localized or diffused, has been held to be a strong indication for hysterectomy, but the difficulty lies in making a diagnosis. I have found diffuse suppuration of the uterine wall in three cases postmortem, but have never detected it during life. With localized abscess my experience has been rather more fortunate: one was recognized only after death, but in three others recovery followed operation. In the first, the symptoms were sudden, and suggested perforation of some hollow viscus. Laparotomy was at once performed, and I found general peritonitis, with effusion of turbid fluid. The cause of this was found to be a ruptured abscess about the size of a walnut in the uterine wall just at the left cornu. After scraping and disinfecting the cavity, I removed the tube on the left side, and folded the broad ligament over the site of the abscess, securing it in position with a double row of catgut sutures. The patient made an uninterrupted recovery, and has since boine a healthy child.

In the next case, a provisional diagnosis of pelvic cellulitis with pyosalpiax was made, and the abdomen opened. It was then apparent that the whole of the posterior surface of the uterus was studded with small abscesses. There was extreme swelling of both broad ligaments, and hysterectomy seemed impossible: the tubes were inflamed, but not suppurating. I closed the abdominal wound, and then incised the posterior cul de sac through the vagina, stripping up the peritoneum from the back of the uterus as high up as possible, the cavity being then packed with gauze. Free discharge of pus followed, and the patient made a good recovery.

In the last case, laparotomy was performed, and extensive pelvic peritonitis was found with a pyosalpinx on the right side, and many 
intestinal adhesions to the uterus and adnexa. After separating these and dealing with the suppurating tube, two subperitoneal abscesses were found in the substance of the muscle just internal to the left cornu. Owing to the thickness of the inflamed broad ligaments, hysterectomy seemed out of the question: the abdominal wound was closed, and the cavity, into which there had been free oozing, was drained. The posterior cul de sac was then opened, and the peritoneum stripped up as before, and a large packing of gauze inserted. Rapid improvement followed, and the patient is now convalescent.

With these cases in view, I cannot but think that, even if suppuration be present in the uterus itself, hysterectomy is not necessarily indicated. It would seem to he better to deal with the condition from the vagina by providing an outlet for the discharge outside the peritoneum. It is, to my mind, far more likely that hysterectomy is really indicated when the uterus does not display any very gross lesion, but, as I have said, the difficulty lies in the selection of the case.

The next point to which I would refer is the utility of bactericidal sera in the streptococcal cases. Some five years ago I used to give at least $50 \mathrm{cc}$, and more often $100 \mathrm{cc}$., of polyvalent antistreptococcic serum to almost every patient suffering from severe streptococcal infection. It soon became evident, however, that these sera were almost useless except in one type of case, viz., where the toxamia was intense, while there was but a slight (evident) local lesion to account for it. The majority of these had streptococei in the circulating blood.

I never saw any harm result, except an urticarial eruption, from the use of these sera, but there seemed to be no reason for their administration where the local lesion was extensive, and on the whole the sera did not come up to the expectation one had formed of them. Some three years ago, however, I became impressed with the value of massive subcutaneous injections of normal saline solution in puerperal infection generally. This impression has deepened with added experience, and consequently almost every case now receives saline subcutaneously for the first few days, and in the most severe cases it is given intravenously also. For the last year, however, in the worst cases, whether the local lesion be extensive or not, I have been adding $50 \mathrm{co}$. of polyvalent antistreptococcic serum to the intravenous saline, and there can be little doubt, I think, firstly, that the saline with the bactericidal serum acts better than the saline alone, and, secondly, that when given with saline, the serum seems to act on cases (with extensive local lesion) in which it is powerless alone. The explanation of this I take to be that the serum contains an immune body, but no complement, and that the saline injection by producing a lencocytosis, supplies the complement required. It is a pity that 
the reputation of these sera has suffered so much from the unscientific way in which small doses of $10 \mathrm{cc}$. have been administered, and the improvement or reverse which followed attributed to the serum alone, the isolated cases being then promptly published in widely-read medical journals. Moreover, the serum employed has often been either monovalent, or stale, or both.

It is much to be wished, however, that the composition of these antistreptococcal sera could be investigated by an independent committee, and the wheat separated from the chaff. The findings of the American Committee are now quite out of date. 